\title{
Custom-made intercalary endoprosthetic reconstruction for a parosteal osteosarcoma of the femoral diaphysis: A case report
}

\author{
SHI-CHANG ZHAO ${ }^{1}$, CHANG-QING ZHANG ${ }^{1}$ and CHUN-LIN ZHANG ${ }^{2}$ \\ ${ }^{1}$ Department of Orthopaedics, Shanghai Sixth People's Hospital, Shanghai Jiaotong University, Shanghai 200233; \\ ${ }^{2}$ Department of Orthopaedics, Shanghai Tenth People's Hospital, Tongji University, Shanghai 200072, P.R. China
}

Received November 10, 2014; Accepted July 30, 2015

DOI: 10.3892/ol.2015.3627

\begin{abstract}
The present report describes a case of a 44-year-old female patient who presented with a palpable mass of the left thigh. A diagnosis of parosteal osteosarcoma (POS) at the femoral diaphysis was made following a diagnostic workup. Previous reports of long bone diaphyseal POS are rare. A long diaphyseal segment of the femur containing the tumor was resected along with a healthy margin of soft tissues, and the damaged bone was reconstructed with a custom-made intercalary endoprosthesis. Subsequent pathological examination of the surgical sample confirmed the diagnosis of POS. No local recurrence or distant metastasis was observed, and the patient had a positive Musculoskeletal Tumor Society score of 28/30 (93.3\%) at the 28-month post-surgery follow-up. The present study describes the clinical, radiological, and pathological features of this rare type of osteosarcoma.
\end{abstract}

\section{Introduction}

Parosteal osteosarcoma (POS) is a rare, slow-growing malignant tumor that predominantly occurs on the surface of the metaphysis of long bones. POS accounts for $\sim 1-2 \%$ of primary malignant bone tumors (1), 4-6\% of all osteosarcomas (2) and $70 \%$ of surface osteosarcomas, with a slight female predilection and a peak incidence in the third and fourth decades of life (1). Unlike conventional osteosarcomas, POS is typically a well differentiated low-grade lesion that has a low tendency to metastasize and has a better prognosis $(1,3,4)$. Chemotherapy or radiotherapy are not recommended for treating cases of POS that do not exhibit dedifferentiation.

The most commonly involved sites of POS include the distal femur, proximal tibia and proximal humerus. Approximately $90 \%$ of POS cases involve the metaphysis and $\sim 67 \%$ are

Correspondence to: Mr. Chun-Lin Zhang, Department of Orthopaedics, Shanghai Tenth People's Hospital, Tongji University, 301 Yanchang Zhong Road, Shanghai 200072, P.R. China E-mail: shzhangchunlin@gmail.com

Key words: parosteal osteosarcoma, femoral diaphysis, wide resection, custom made intercalary endoprosthesis confined to the metaphysis. The posterior aspect of the distal femur accounts for $\sim 70 \%$ of all cases (5), and furthermore, diaphyseal involvement is observed in $<10 \%$ of all cases (6-8).

Wide resection of POS is recommended to prevent local recurrence and the rate of distant metastasis; however, the optimal technique for long bone diaphyseal reconstruction following tumor resection is undecided. There are a number of techniques available for femoral reconstruction of diaphyseal defects following excision of bone tumors. These include the use of autogenous vascularized fibular grafts $(9,10)$, segmental allografts (11-13), autogenous extracorporeally irradiated bone $(14,15)$, distraction osteogenesis $(16,17)$ and custom-made intercalary endoprostheses (18-20).

The present case report describes the clinical, radiological and pathologic features of a 44-year old patient diagnosed with POS localized in the diaphysis of the left femur. A custom-made intercalary endoprosthesis was used to achieve anatomical and functional reconstruction for a $16 \mathrm{~cm}$ bone defect following en bloc tumor resection. Written informed consent was obtained from the patient and the Ethical Review Board of Shanghai Tenth People's Hospital, Tongji University School of Medicine (Shanghai, China) approved the experimental procedures.

\section{Case report}

A 44-year-old female was admitted to Shanghai Tenth People's Hospital (Shanghai, China) on December 22, 2011 for treatment of a left femoral diaphyseal lesion. The patient had no history of trauma or infection. Physical examination revealed a well-defined, firm, painless mass in the patient's medial thigh, and the patient had full active range of motion of the hip and knee joint. There was no vascular or neurological deficit associated with the affected limp. Routine blood investigations were not relevant for any of the pathological findings.

Plain radiography demonstrated a densely ossified mass with a sessile configuration attached to the surface of the left femur diaphysis. No radiolucent cleft separated the mass from the host bone (Fig. 1A and B) and computed tomography (CT) demonstrated an intact and continuous femoral cortex encircling the mass (Fig. 2A-C). Small unmineralized areas were observed predominantly at the periphery of the lesion. Axial view $\mathrm{CT}$ scans demonstrated that the mass had infiltrated and destroyed the cortical bone, but had not invaded the medullary cavity (Fig. 2D). Magnetic resonance imaging (MRI) revealed 
a tumor on the surface of the femoral diaphysis that measured $10 \times 5.5 \times 4.5 \mathrm{~cm}$ and displayed low-to-intermediate signal intensity (SI), when compared to adjacent muscles on T1-weighted (T1W) images (Fig. 3A). T2-weighted images displayed a heterogeneous lesion, containing areas of low-to-intermediate SI mixed with peripheral hyperintense areas (Fig. 3B). Furthermore, T2W images demonstrated no invasion of the medullar canal by the tumor, which was initially indicated on CT scan. Subsequent CT scans of the chest and whole-body nuclear scans revealed no metastases or other bony abnormalities.

Following the CT and MRI scans, an incisional biopsy was performed. The samples were fixed in $10 \%$ formaldehyde, decalcified in EDTA (Sinopharm Chemical Reagent Co. Ltd., Shanghai, China), dehydrated in a graded series of ethanol (70-100\%) and embedded in paraffin. Sections were cut to a thickness of $5 \mu \mathrm{m}$ in the longitudinal direction of the tibia, then stained with hematoxylin and eosin (H\&E) and observed using transmitted light microscopy at a magnification of x100 (DMI6000B; Leica Biosystems, Wetzlar, Germany). Histologically, the lesion exhibited moderate cellular malignant tumor proliferation, with slightly atypical and rarely mitotic fusiform cells surrounded by osteoid trabeculae. These observations led to a diagnosis of POS (Fig. 4).

Preoperatively, a custom-made prosthesis made of titanium alloy (Ti6A14V), with precisely controlled architecture for femoral diaphysis defects, was fabricated using computer assisted design, based on plain radiographs and CT/MRI three dimensional data files of the patient (Fig. 5). The prosthesis was designed with an intramedullary stem at each end, these allow insertion into the corresponding femoral canal. Both stems are screwed to provide rotational stability, and fixation may be further enhanced with the use of extracortical plates.

The surgical approach followed general principles of malignant bone tumor surgery. The patient was positioned supine, and the standard lateral approach to the femur was used, with the incision extending from the lateral knee joint line proximally to the greater trochanter. A 2-cm cuff of healthy tissue was left with the tumor, and the biopsy track was left in continuity with the specimen. The femoral vessels were dissected from proximal to distal and small perforating branches were ligated as they enter the femur. The sciatic nerve was located and protected. The femur was osteotomized (proximally) $5 \mathrm{~cm}$ below the greater trochanter and (distally) $15 \mathrm{~cm}$ above the knee. The resected tumor was removed and specimens of the tumor margins were sent for histologic evaluation. The resection length measured $16 \mathrm{~cm}$ (Fig. 6). The frozen sections of the marrow at the site of proximal and distal resections were reviewed to ensure the margins were negative for tumor cells before reconstruction. The femoral diaphysis was then reconstructed using the custom-made intercalary endoprosthesis (Fig. 7). The patient received prophylactic antibiotics (cefotiam, $2.0 \mathrm{~g}$ ) twice a day for 3 days. Active physiotherapy commenced on the second day following the operation, and the patient was able to support full weight on the limb by the time of discharge. The patient recovered and returned to preoperative life. No local recurrence or metastasis was observed. The range of motion of the hip and knee was not restricted, and the patient had a good Musculoskeletal Tumour Society (MSTS) score (21) of 28/30 (93.3\%) at the most recent 28-month follow-up.
A

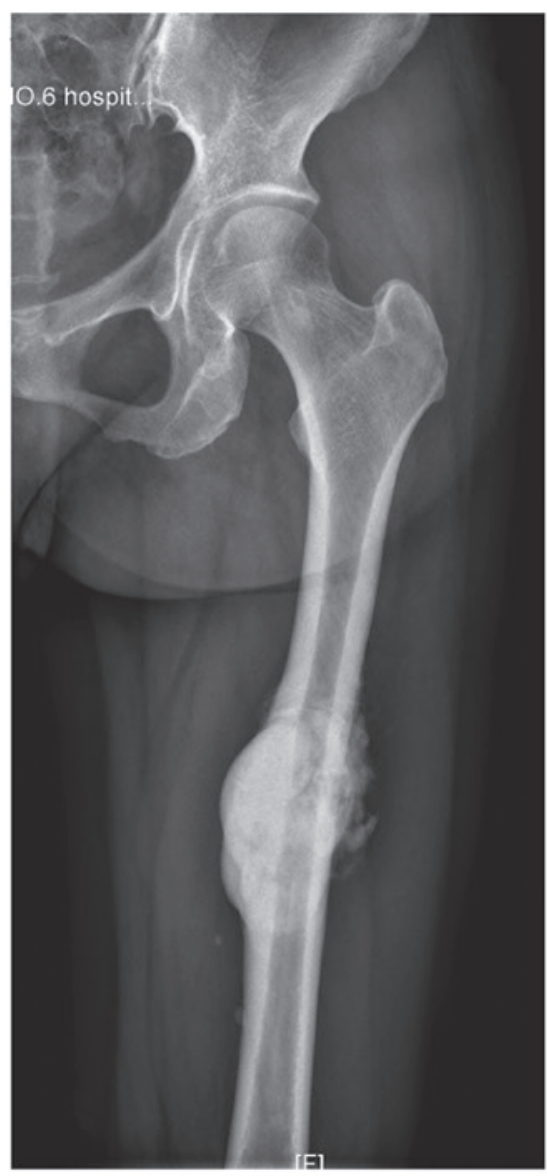

B

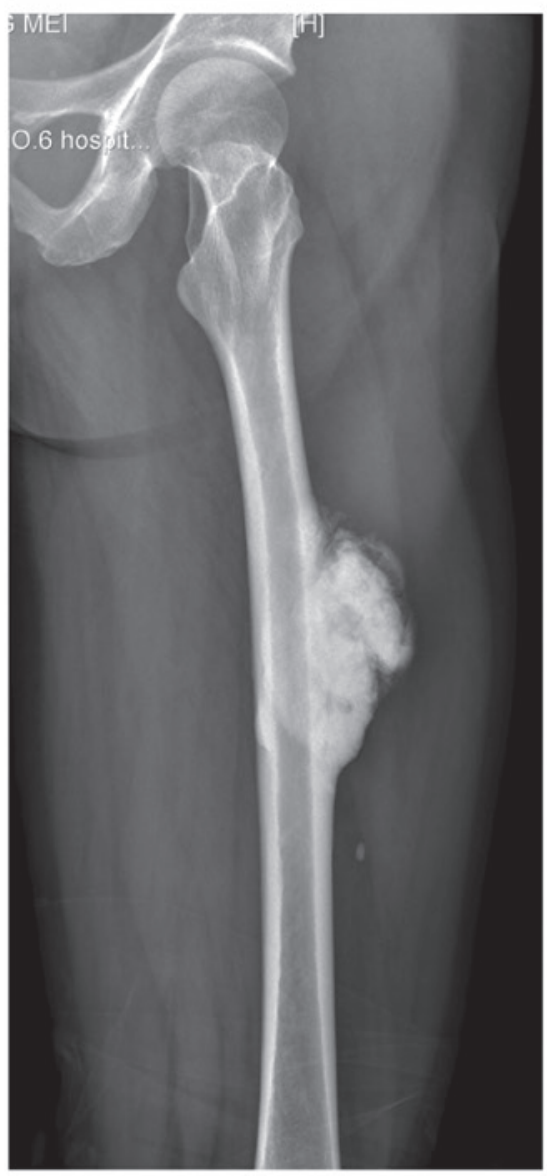

Figure 1. (A) Anterioposterior and (B) lateral radiographs of the left femur demonstrate a densely ossified juxtacortical lesion, adjacent to the anterior aspect of the left femoral diaphysis. 


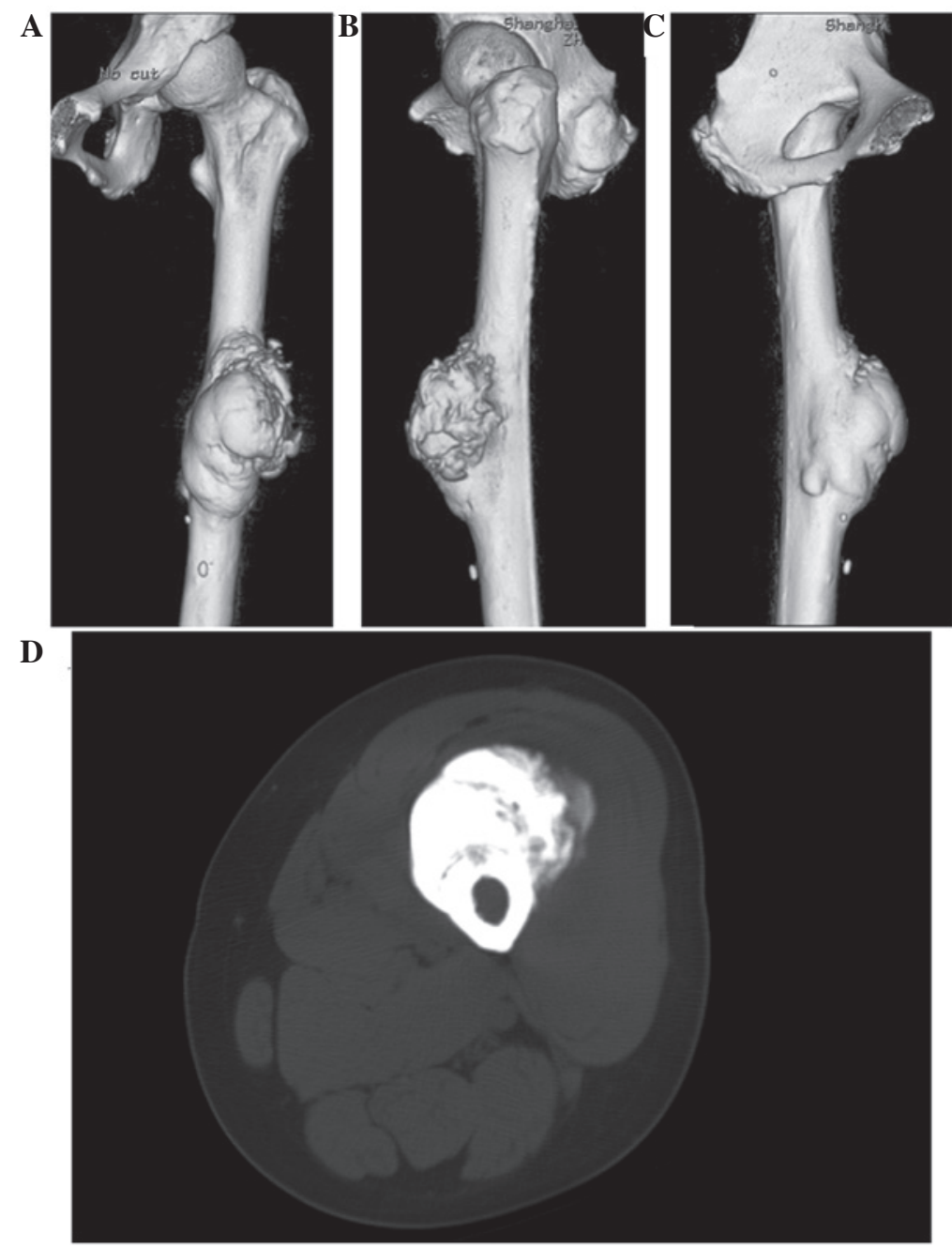

Figure 2. (A-C) Three dimensional computed tomography (CT) scans of the left femur demonstrate a broad-based, mineralized lesion arising from the surface of the femoral diaphysis. (D) An axial view CT scan reveals the tumor has damaged the cortical bone, but has not invaded the medullary cavity of the left femur.
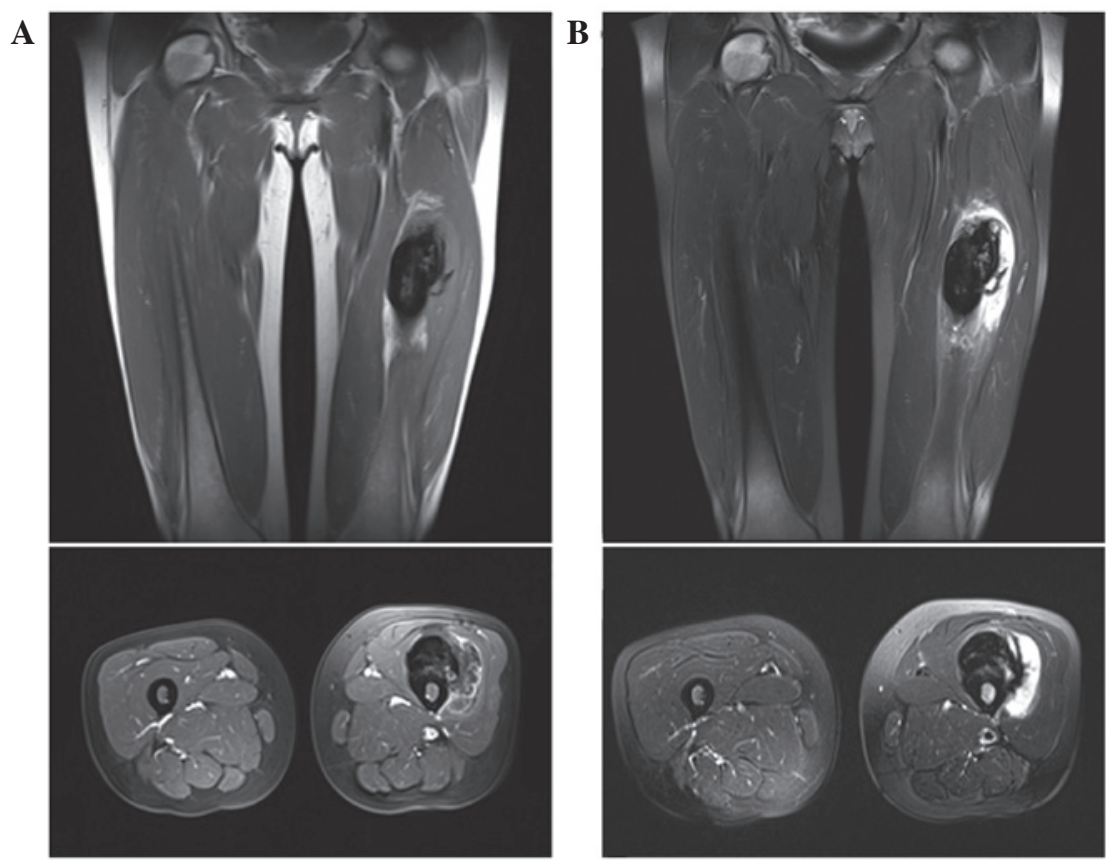

Figure 3. (A) T1-weighted coronal and axial MR images demonstrating a round, low-to-intermediate signal intensity tumor, originating from the anterior aspect of the femoral diaphysis, measuring $10 \times 5.5 \times 4.5 \mathrm{~cm}$. (B) T2-weighted coronal and axial MR images reveal a heterogeneous lesion, with areas of low-to-intermediate signal intensity mixed with hyperintense areas. 


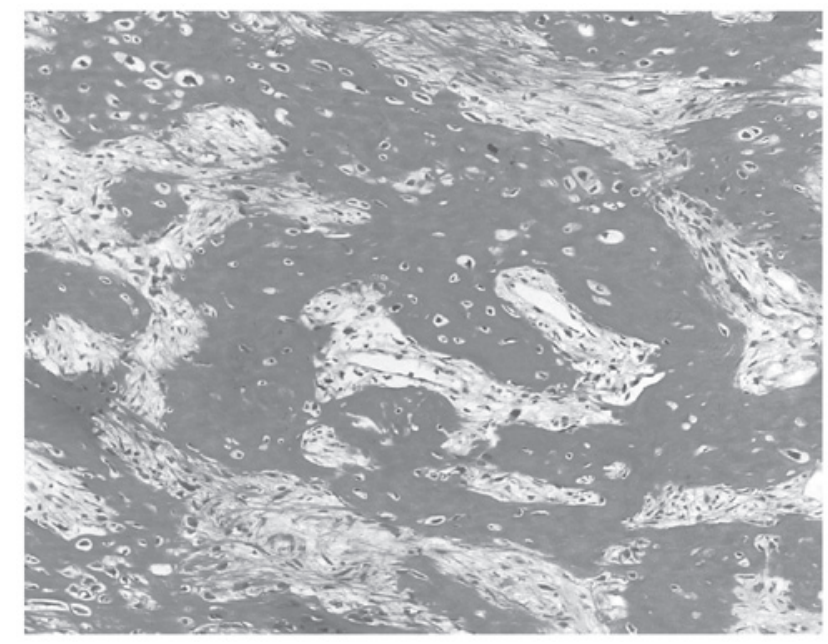

Figure 4. Histopathological analysis of the tumor biopsy revealed well-formed osseous trabeculae surrounded by spindle cells, with minimal nuclear atypia and little to no pleomorphism. Hematoxylin and eosin; magnification, x100.

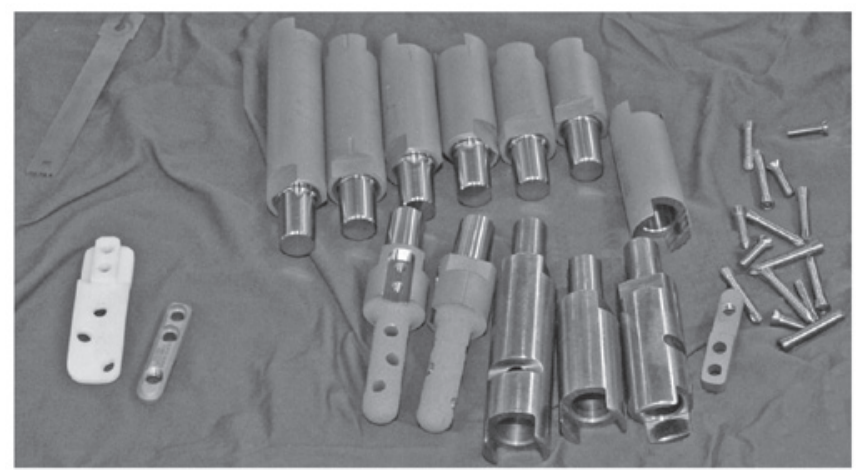

Figure 5. Components of the custom-made intercalary femoral diaphyseal endoprosthesis.

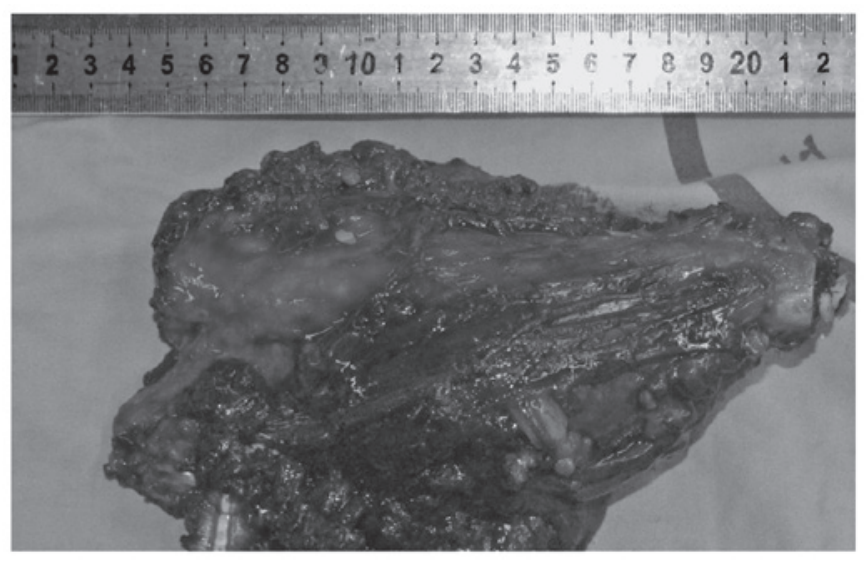

Figure 6. Macroscopic appearance of the 16-cm excised specimen. A large tumorous mass measuring $\sim 10 \mathrm{~cm}$ was located on the surface of the femoral diaphysis.

\section{Discussion}

First described in 1951 by Geschickter and Copeland (22), POS is a rare, slowly progressive tumor that is typically reported in the metaphyseal of the long bones, rarely affecting the diaphysis (7,23). Clinically, POS presents as a hard, immobile swelling with no or slight pain. The symptoms are often of prolonged duration (4). Pulmonary metastases occur late in the course of the disease, typically following one or more local relapses. Therefore, surgical intervention focuses on the local control of the primary tumor (7). Early recognition depends on clinical suspicion and a precise radiological and histological evaluation.

Radiologically, the classic appearance of POS is that of a heterogeneous, amorphous, and densely mineralized lesion, arising from the subjacent cortical bone surface and extending into the soft tissues (2). A characteristic finding is a radiolucent line, separating the dense bony mass of the tumor from the host bone, with the exception of the site of attachment. This is called the cleavage plane, and represents the uncalcified thickened periosteum. However, disappearance of the cleavage plane and the preservation of a continuous femoral cortex encircling the tumor, as observed in the present case, are probably attributed to the gradual destruction of the cortical bone by the circumferential growth of the lesion $(3,24)$. Occasionally, tumors may completely encompass the involved bone, and the base of the lesion is typically more ossified than the outermost portion. CT scans can accurately define the extent of the tumor and cortical integrity (7); however, they may not reveal small satellite nodules beyond the main tumor (25). MRI images vary in relation to tumor size, in addition to the presence of dense osteoid, cartilage, hemorrhage, necrosis or areas of high-grade tumor or dedifferentiation. Generally, POS are hypointense on both T1W and T2W MR images, particularly when the tumors are small. Jelinek et al (3) propose that low signal intensity on both T1W and T2W MR images indicates a low-grade lesion, and high signal intensity on T2-weighted images indicates a high-grade component. MRI is optimal for identifying the appropriate biopsy site and potential medullary invasion prior to surgical intervention (7). MRI and CT scans are useful in detecting characteristics of tumor aggressiveness, such as cortical disruption and intramedullary or soft tissue expansion. POS does not typically invade the medullary cavity unless the lesion is of long duration or the patient has received surgical treatment. Approximately $10-20 \%$ of patients with POS demonstrate medullary involvement $(23,25)$.

Histological analysis of POS typically reveals long, narrow trabeculae or ill-defined islands of osteoid and woven bone, separated by a fibrous stroma. The trabeculae may undergo maturation that results in the formation of normal-appearing lamellar bone with minimal cytological atypia and only rare mitotic activity $(1,2)$. There is typically minimal to no involvement of the underlying medullary bone, and the cortex is often thickened and deformed. These features were exhibited by the presenting lesion of the patient in the present case, strongly indicating the diagnosis of POS.

POS is relatively easy to diagnose according to the imaging and the biopsy results, with the exception of advanced cases that exhibit medullary involvement or dedifferentiation. Radiologically, POS should be differentiated from periosteal osteosarcoma, mature juxtacortical myositis ossificans, exostosis, osteoma, Ewing's sarcoma, and periosteal chondrosarcoma $(26,27)$. Histopathologically, POS must be differentiated from various conditions, including myositis ossificance adjacent to cortical bone, and periosteal 
A

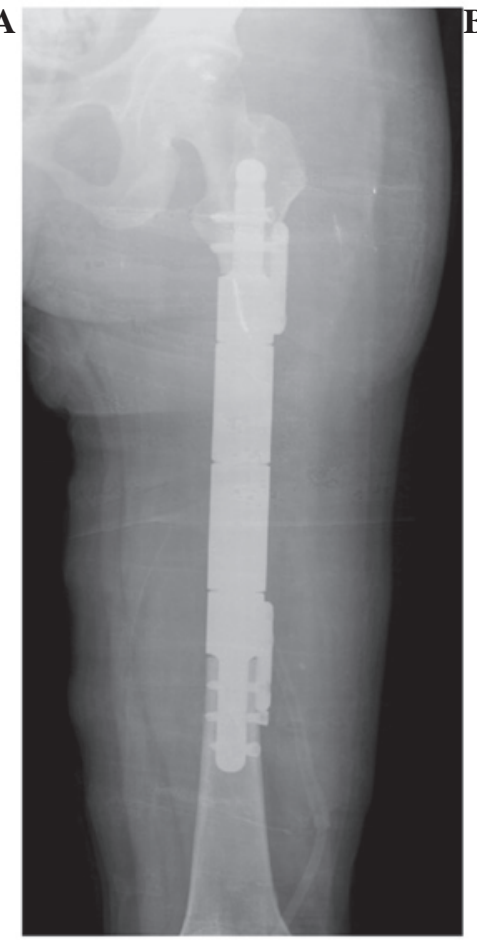

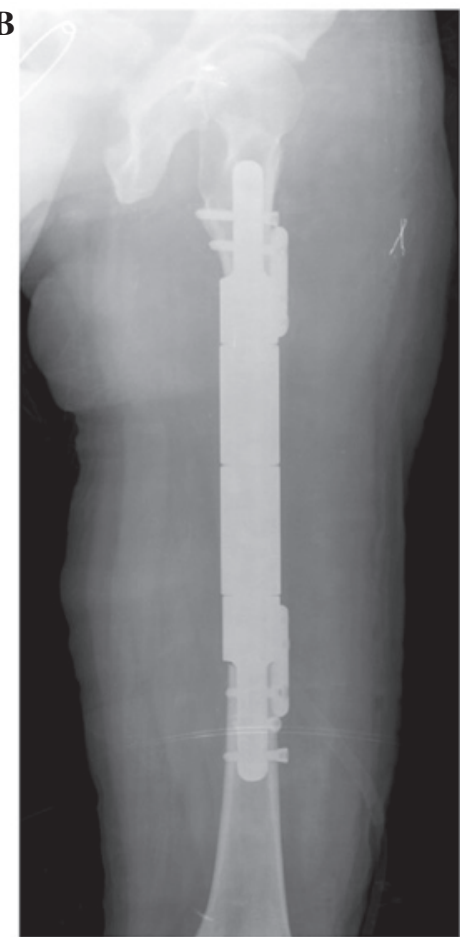

Figure 7. Post-operative (A) anterioposterior and (B) lateral radiographs demonstrating the reconstruction of the bone defect following tumor resection, using an intercalary custom-made endoprosthesis.

chondrosarcoma. A misdiagnosis with these conditions may lead to resection with an inadequate surgical margin.

Segmental resection of a primary bone sarcoma in the diaphysis is a relatively low-risk method of preventing local recurrence or distant metastasis, and preserves adjacent joints and juxta-articular bone, as well as the epiphysis in children, making it an attractive option for limb salvage. However, the successful reconstruction of segmental intercalary defects following resection of a POS in the femoral diaphysis is difficult to achieve. There are several biological and nonbiological materials available for the reconstruction of diaphyseal bone defects following tumor resection. However, various factors including the ease of, and applicability of the procedure, as well as durability, complications, oncological safety and functional outcome need to be considered.

Vascularized and non-vascularized autogenous fibular grafts are associated with a significant risk of nonunion, fracture and morbidity at the donor site, and are therefore not appropriate for large bone defects, which are particularly common in the femur $(9,13,28)$. It typically takes numerous years until hypertrophy of the graft is sufficient to allow full weight-bearing, which can be a major disadvantage for an individual who wishes to resume normal life, rather than rely on crutches for a prolonged and uncertain period of time. Previously published studies of the reconstruction of femoral defects with autografts demonstrated that the average time to full weight bearing was 19 months, and the incidence of complications is closely associated with the length of the segment replaced (29).

Large-segment allograft reconstruction is an attractive proposal as it allows mechanical and biological reconstruction during the same procedure. However, it is associated with difficulty in obtaining appropriately sized allografts from bone banks and high rates of complications, including fracture, nonunion, transmission of disease and infection (30). To date, complete endosteal revascularization has not been demonstrated $(31,32)$, and it takes 16 months for the diaphyseal allograft to unite, therefore, the patient must remain non- or partially weight bearing during the first few months following the operation (33). An additional study has demonstrated a $50 \%$ loss in strength of the allograft bone after 10 years (34). Loss of strength was correlated with an increase in the prevalence of microfracture and a reduction in bone mineral density within the cortex of the retrieved allograft.

Extracorporeally irradiated bone offers an attractive option for reconstructing diaphyseal defects, by acting as a close-fitting scaffold for creeping substitution and incorporation, but it takes a long time to revascularize and incorporate into surrounding bone $(14,35)$. Although the rates of nonunion and fracture are similar to those of allograft bone, the overall rate of complication is $<52 \%(15,35,36)$. Furthermore, the procedure is not applicable for bones that are structurally weak and in bones with pathological fractures.

Distraction osteogenesis (DO) using the Ilizarov method provides bone with adequate biomechanical strength, and it is useful in the treatment of post-traumatic segmental bone defects. However, this method is time-consuming and associated with a high incidence of complications (37). Tsuchiya et al $(16,17)$ were the first to introduce limb-salvage surgery using DO combined with conventional bone transport and the application of an external fixator. In their studies, early weight bearing was allowed, and gradual distraction started 1-2 weeks following the operation, at a rate of $1 \mathrm{~mm}$ per day. Although the reported results were good, the authors advised against using this method for segmental defects exceeding $15 \mathrm{~cm}$ in length, rendering the technique inappropriate for larger femoral tumors. 
Endoprosthetic replacements of the femoral diaphysis have demonstrated an acceptable postoperative complication rate, long-term survival and functional outcome, following resection of primary malignant bone tumors $(19,38)$. The complications encountered during this procedure include infection $(39,40)$, aseptic loosening, mechanical failure, fracture either of the prosthesis or of adjacent bone (19,39-41), local recurrence and metastatic spread (38). Compared with other reconstructive options, the use of a custom-made intercalary endoprosthesis for the reconstruction of the femoral diaphysis has some advantages. These include a short operating time and hospital stay, with a low incidence of early complications and reduced risk of disease transmission. Furthermore, it allows early patient mobility (without walking aids) and immediate commencement of post-operative adjuvant therapy. Patients are able to return to a more or less normal life much earlier (12). Hanna et al (38) reported the mean MSTS score for the patients retaining their diaphyseal endoprosthesis for 10 years following surgery was $87 \%$ (range, $67-93 \%$ ). In the present study, the patient returned to preoperative life with full range of hip and knee joint motion and an MSTS score of $28 / 30(93.3 \%)$ at the 18-month post-surgery follow-up.

The femoral diaphyseal location in the present POS case is uncommon, within an already extremely rare tumor entity. An intercalary custom-made endoprosthesis was used to reconstruct the damaged bone following tumor resection. No early complications, local recurrence and distant metastasis were observed, and the MSTS score was 28/30 (93.3\%) at the 28 -month post-surgery follow-up. The present authors have described herein the clinical, radiological, and pathological characteristics of POS and have discussed its surgical management.

\section{References}

1. Murphey MD, Robbin MR, McRae GA, Flemming DJ, Temple HT and Kransdorf MJ: The many faces of osteosarcoma. Radiographics 17: 1205-1231, 1997.

2. Azura M, Vanel D, Alberghini M, Picci P, Staals E and Mercuri M: Parosteal osteosarcoma dedifferentiating into telangiectatic osteosarcoma: Importance of lytic changes and fluid cavities at imaging. Skeletal Radiol 38: 685-690, 2009.

3. Jelinek JS, Murphey MD, Kransdorf MJ, Shmookler BM, Malawer MM and Hur RC: Parosteal osteosarcoma: Value of MR imaging and CT in the prediction of histologic grade. Radiology 201: 837-842, 1996.

4. Park YK and Ryu KN: Parosteal osteosarcoma of the scapula. J Korean Med Sci 14: 586-588, 1999.

5. Subasi M, Kapukaya A, Buyukbayram H and Bilici A: Unusual benign bone lesion simulating parosteal osteosarcoma. J Orthop Sci 11: 529-532, 2006

6. Partovi S, Logan PM, Janzen DL, O'Connell JX and Connell DG: Low-grade parosteal osteosaracoma of the ulna with dedifferentiation into high-grade osteosarcoma. Skeletal Radiol 25: 497-500, 1996.

7. Dönmez FY, Tüzün U, Başaran C, Tunaci M, Bilgiç B and Acunaş G: MRI findings in parosteal osteosarcoma: Correlation with histopathology. Diagn Interv Radiol 14: 142-152, 2008

8. Haworth JM, Watt I, Park WM and Roylance J: Diaphyseal osteosarcoma. Br J Radiol 54: 932-938, 1981.

9. Chang DW and Weber KL: Use of a vascularized fibula bone flap and intercalary allograft for diaphyseal reconstruction after resection of primary extremity bone sarcomas. Plast Reconstr Surg 116: 1918-1925, 2005.

10. Chen CM, Disa JJ, Lee HY, et al: Reconstruction of extremity long bone defects after sarcoma resection with vascularized fibula flaps: a 10-year review. Plast Reconstr Surg 119: 915-924; discussion 925-916, 2007.
11. Donati D, Di Liddo M, Zavatta M, Manfrini M, Bacci G, Picci P, Capanna R and Mercuri M: Massive bone allograft reconstruction in high-grade osteosarcoma. Clin Orthop Relat Res 377: 186-194, 2000.

12. Muscolo DL, Ayerza MA, Aponte-Tinao L, Ranalletta M and Abalo E: Intercalary femur and tibia segmental allografts provide an acceptable alternative in reconstructing tumor resections. Clin Orthop Relat Res 426: 97-102, 2004.

13. Ogilvie CM, Crawford EA, Hosalkar HS, King JJ and Lackman RD: Long-term results for limb salvage with osteoarticular allograft reconstruction. Clin Orthop Relat Res 467: 2685-2690, 2009.

14. Chen TH, Chen WM and Huang CK: Reconstruction after intercalary resection of malignant bone tumours: Comparison between segmental allograft and extracorporeally-irradiated autograft. J Bone Joint Surg Br 87: 704-709, 2005.

15. Krieg AH, Davidson AW and Stalley PD: Intercalary femoral reconstruction with extracorporeal irradiated autogenous bone graft in limb-salvage surgery. J Bone Joint Surg Br 89: 366-371, 2007.

16. Tsuchiya H, Tomita K, Minematsu K, Mori Y, Asada N and Kitano S: Limb salvage using distraction osteogenesis. A classification of the technique. J Bone Joint Surg Br 79: 403-411, 1997.

17. Tsuchiya H, Wan SL, Sakayama K, Yamamoto N, Nishida H and Tomita K: Reconstruction using an autograft containing tumour treated by liquid nitrogen. J Bone Joint Surg Br 87: 218-225, 2005.

18. Abudu A, Carter SR and Grimer RJ: The outcome and functional results of diaphyseal endoprostheses after tumour excision. J Bone Joint Surg Br 78: 652-657, 1996.

19. Aldlyami E, Abudu A, Grimer RJ, Carter SR and Tillman RM: Endoprosthetic replacement of diaphyseal bone defects. Long-term results. Int Orthop 29: 25-29, 2005.

20. Ahlmann ER and Menendez LR: Intercalary endoprosthetic reconstruction for diaphyseal bone tumours. J Bone Joint Surg Br 88: 1487-1491, 2006.

21. Enneking WF, Dunham W, Gebhardt MC, Malawar MM and Pritchard DJ: A system for the functional evaluation of reconstructive procedures after surgical treatment of tumors of the musculoskeletal system. Clin Orthop Relat Res: 241-246, 1993.

22. Geschickter CF and Copeland MM: Parosteal osteoma of bone: A new entity. Ann Surg 133: 790-807, 1951.

23. Okada K, Frassica FJ, Sim FH, Beabout JW, Bond JR and Unni KK: Parosteal osteosarcoma. A clinicopathological study. J Bone Joint Surg Am 76: 366-378, 1994.

24. Suresh S and Saifuddin A: Radiological appearances of appendicular osteosarcoma: A comprehensive pictorial review. Clin Radiol 62: 314-323, 2007.

25. Hudson TM, Springfield DS, Benjamin M, Bertoni F and Present DA: Computed tomography of parosteal osteosarcoma. AJR Am J Roentgenol 144: 961-965, 1985.

26. Uyttendaele D, De Schryver A, Claessens H, Roels H, Berkvens P and Mondelaers W: Limb conservation in primary bone tumours by resection, extracorporeal irradiation and re-implantation. J Bone Joint Surg Br 70: 348-353, 1988.

27. Lindell MM Jr, Shirkhoda A, Raymond AK, Murray JA and Harle TS: Parosteal osteosarcoma: Radiologic-pathologic correlation with emphasis on CT. AJR Am J Roentgenol 148: 323-328, 1987.

28. Rose PS, Shin AY, Bishop AT, Moran SL and Sim FH: Vascularized free fibula transfer for oncologic reconstruction of the humerus. Clin Orthop Relat Res 438: 80-84, 2005.

29. Han CS, Wood MB, Bishop AT and Cooney WP III: Vascularized bone transfer. J Bone Joint Surg Am 74: 1441-1449, 1992.

30. Ozaki T, Hillmann A, Bettin D, Wuisman P and Winkelmann W: Intramedullary, antibiotic-loaded cemented, massive allografts for skeletal reconstruction. 26 cases compared with 19 uncemented allografts. Acta Orthop Scand 68: 387-391, 1997.

31. Brown KL: Limb reconstruction with vascularized fibular grafts after bone tumor resection. Clin Orthop Relat Res 262: 64-73, 1991.

32. Kattapuram SV, Rosol MS, Rosenthal DI, Palmer WE and Mankin HJ: Magnetic resonance imaging features of allografts. Skeletal Radiol 28: 383-389, 1999.

33. San Julian Aranguren M, Leyes M, Mora G and Cañadell J: Consolidation of massive bone allografts in limb-preserving operations for bone tumours. Int Orthop 19: 377-382, 1995.

34. Fox EJ, Hau MA, Gebhardt MC, Hornicek FJ, Tomford WW and Mankin HJ: Long-term followup of proximal femoral allografts. Clin Orthop Relat Res 397: 106-113, 2002. 
35. Amitani A, Yamazaki T, Sonoda J, Tanaka M, Hirata H, Katoh K and Uchida A: Preservation of the knee joint in limb salvage of osteosarcoma in the proximal tibia. A case report. Int Orthop 22: 330-334, 1998

36. Fuchs B, Ossendorf C, Leerapun T and Sim FH: Intercalary segmental reconstruction after bone tumor resection. Eur J Surg Oncol 34: 1271-1276, 2008.

37. Hornicek FJ, Gebhardt MC, Tomford WW, Sorger JI, Zavatta M, Menzner JP and Mankin HJ: Factors affecting nonunion of the allograft-host junction. Clin Orthop Relat Res 382: 87-98, 2001.

38. Hanna SA, Sewell MD, Aston WJ, Pollock RC, Skinner JA, Cannon SR and Briggs TW: Femoral diaphyseal endoprosthetic reconstruction after segmental resection of primary bone tumours. J Bone Joint Surg Br 92: 867-874, 2010.
39. Guo W, Ji T, Yang R, Tang X and Yang Y: Endoprosthetic replacement for primary tumours around the knee: Experience from Peking University. J Bone Joint Surg Br 90: 1084-1089, 2008.

40. Gosheger G, Gebert C, Ahrens H, Streitbuerger A, Winkelmann W and Hardes J: Endoprosthetic reconstruction in 250 patients with sarcoma. Clin Orthop Relat Res 450: 164-171, 2006.

41. Sewell MD, Spiegelberg BG, Hanna SA, Aston WJ, Bartlett W, Blunn GW, David LA, Cannon SR and Briggs TW: Total femoral endoprosthetic replacement following excision of bone tumours. J Bone Joint Surg Br 91: 1513-1520, 2009. 